\title{
Cardiac PET and PET/CT Imaging
}

\author{
M. F. Di Carli and M. J. Lipton, eds.
}

New York, NY: Springer, 2007, 466 pages, \$149

Cardiac PET and PET/CT Imaging is a comprehensive, informative, up-to-date, state-of-the-art textbook on newer cardiac imaging technologies and also provides valuable insight into the future of cardiac imaging. The book has 6 parts; the first 5 parts or sections are subdivided into several chapters that cover a wide spectrum of presently available newer cardiac imaging modalities and future research goals. The first 3 parts progress in a systematic and orderly manner, starting with the basics of PET instrumentation, the principles of PET, PET tracers and radiopharmaceuticals, CT cardiac anatomy, iodinated contrast agents, radiation safety, patient preparation, stress protocols, PET and PET/CT protocols, myocardial PET interpretation, and PET quantification for assessing preclinical coronary artery disease. The relative merits of coronary $\mathrm{CT}$ angiography and MRI along with integrated assessment of myocardial perfusion using coronary angiography and PET/CT for diagnosing and managing coronary artery disease are clearly and well presented.

Part 4 discusses various diagnostic approaches to heart failure, including myocardial metabolism and viability using PET and PET/CT and assessment of myocardial perfusion, viability, and function with contrast CT and MRI, and compares different imaging modalities for assessing myocardial viability. The chapter on the role of imaging in heart failure summarizes currently available information on the role and usefulness of cardiac PET of the autonomic innervations of the failing heart.

Part 5 discusses the emerging and future potential role of molecular imaging in characterizing plaques and evaluating high-risk vulnerable plaques with correlative PET/CT and MRI; a basic knowledge of these topics will be essential to better understand future advances and developments in this field. Chapter 25 discusses the emerging useful potential of PET and PET/CT in monitoring gene and cardiac cellular therapy. Chapter 26 explains the mechanism of angiogenesis and molecular imaging of angiogenesis using targeted and nontargeted approaches both at the preclinical development stages and in clinical applications so as to better identify and differentiate a responder to angiogenesis therapies from a nonresponder. Targeted angiogenesis imaging in conjunction with standard imaging based on physiologic parameters - the so-called hybrid imaging approach-will play an increasingly useful and vital future role, with diagnostic and prognostic implications in evaluating gene

COPYRIGHT (c) 2008 by the Society of Nuclear Medicine, Inc. DOI: 10.2967/jnumed.108.050609 therapy. Chapters 17, 18, and 21 are a must-read for clinical PET, whereas chapters 23 through 26 are informative and useful regarding ongoing research and will help one keep up with future developments in this evolving field.

Part 6 comprises multiple case presentations with good illustrations and good explanations of image findings, and many are supported with additional relevant correlations or follow-up findings. There are 18 diverse illustrative cases, including normal study findings, misregistration and attenuation correction artifacts, incidental findings, abnormal high-risk PET/CT scans, myocardial viability, integrated myocardial perfusion with assessment of coronary artery calcium score, left ventricular function assessment, and CT coronary angiography. The writing is clear and understandable; even difficult topics such as physics, radiochemistry, and biochemistry are well presented and are understandable with concentrated reading. An understanding of these necessary basic prerequisites along with the pathophysiology of the diseased heart is essential to an understanding of molecular imaging.

Brief summary points at the end of each chapter highlight and underscore key points. Most illustrations and figures are in color; all are of high quality, and many include arrows delineating the abnormalities. Tables and other nonimage figures are well displayed, relevant, and easy to understand. References range from adequate to more than adequate, except for chapter 17 , relating to cardiac anatomy on CT, which did not have any reference. Paper quality is excellent, with a glossy finish; the hardcover book is well bound and will stand up well to repeated, frequent handling.

Limitations are few; the wide left margin on each page of text, comprising almost one third of the entire page, wastes space. Better centering of the text with reduction of the margin would have made for a slightly slimmer and more eco-friendly textbook. Figure 21.7 , on page 321 , mentions nonexistent red and blue arrows. The arrows are instead represented as differing shades of gray in a black and white image.

Drs. Marcelo F. Di Carli and Martin J. Lipton and their talented team of authors are to be congratulated on doing a phenomenal job resulting in this informative, educational, and much-needed textbook on cardiac PET and PET/CT. Unlike the recent explosion of textbooks on oncologic uses of PET, PET/CT, and molecular imaging, there are few comprehensive or reference-quality textbooks on the newly emerging field of cardiac uses of these techniques - a field that is still in its infancy. This book is a trailblazer that will 
help advance the field of cardiac PET. I found the book to be useful, exciting, and helpful in advancing my knowledge of this important new direction in nuclear cardiology. Many chapters are a must-read, and in fact, the book is a must-have for cardiologists, nuclear medicine physicians, and radiologists involved in cardiac imaging and is useful for readers at all levels, from residents to fellows to medical practitioners.

I highly recommend this book to anyone who is interested in keeping up with the dynamic changes in the field of cardiac PET, PET/CT, and molecular imaging. The book will be a useful addition to medical and hospital libraries. We have arrived at a crossroads in nuclear cardiology and are forging a new and exciting path in largely uncharted territory that will certainly influence the way we soon will be imaging the heart in disease states. The goal of future research is to help physicians customize or tailor regular or highly specialized therapies in the most cost-efficient, highly effective way for individual patients based on a sound understanding of the pathophysiology, pharmacokinetics, functional aspects, and biologic aspects of gene therapy and cellular delivery using selective molecular imaging.

Usha A. Joseph

University of Texas Medical School Houston, Texas 